\title{
Escala de atitudes relacionadas às competências odontológicas: desenvolvimento e validação
}

\author{
Manoela Almeida Santos da Figueira*; Paulo Savio Angeiras de Goes**; Monica Cristina Batista de \\ Melo***; Maria da Conceição do Nascimento****; Silvia Regina Jamelli**; Gustavo Pina Godoy \\ * Coordenadora do Curso de Odontologia, Faculdade \\ Pernambucana de Saúde \\ ** Professor Adjunto, Departamento de Clínica e Odontologia \\ Preventiva, Universidade Federal de Pernambuco \\ *** Professora. Programa de Mestrado Profissional em Educação \\ para Área de Saúde, Faculdade Pernambucana de Saúde \\ **** Cirurgiã-dentista, Universidade Federal de Pernambuco \\ ***** Professor Auxiliar, Departamento de Patologia, Universidade \\ Federal de Pernambuco
}

Recebido em 24/10/2019. Aprovado em 08/04/2020.

\begin{abstract}
RESUMO
O objetivo do presente estudo foi desenvolver e realizar a validação de conteúdo e de face de uma escala de atitudes relacionadas às competências odontológicas (EA-CO). Tratou-se de estudo de desenvolvimento metodológico envolvendo construção e validação de uma escala, com abordagem quanti e qualitativa. O referencial teórico para o construto da escala envolveu o conceito de atitude no campo da Psicologia Social, e o de competência, no campo da Educação, qualificada pelas Diretrizes Curriculares Nacionais para os cursos de Odontologia, originando os indicadores: Empatia na relação profissional-paciente, Aprendizagem ao longo da vida, Disponibilidade para aprendizagem interprofissional, Proteção aos direitos do paciente, Responsabilidade social e Inteligência emocional. Dez especialistas participaram da validação de face da matriz de indicadores (Técnica do Grupo Nominal) e dos itens (Questionário) e a versão preliminar foi testada em piloto com 17 estudantes de Odontologia. Houve consenso para permanência dos indicadores, exceto para Inteligência emocional. A escala (tipo Likert, 5 pontos) resultou em 53 itens, sendo 13 alterados da versão original. A EACO foi desenvolvida e atendeu aos critérios de validação de conteúdo e de face utilizados no estudo. Descritores: Educação em Odontologia. Atitude do Pessoal de Saúde. Competência Profissional. Avaliação Educacional. Estudos de Validação.
\end{abstract}




\section{INTRODUÇÃO}

Há um grande desafio em avaliar as atitudes e competências de profissionais e em especial das profissões de saúde. No campo social, as atitudes conceituam-se como crenças e sentimentos que predispõem uma ação ${ }^{1}$. No âmbito educacional, as atitudes correspondem um dos elementos-chave para observar o desempenho ${ }^{2}$ inclusive em situação real resultante de aprendizagem complexa e da combinação de habilidades cognitivas, atitudinais e psicomotoras decorrentes de processos mentais de transferência, realizados em diversos contextos, ao longo da vida ${ }^{3-7}$.

A valorização das atitudes na formação do profissional de saúde tem recebido destaque em documentos nacionais ${ }^{7-9}$ e internacionais ${ }^{10,11}$. As Diretrizes Curriculares Nacionais (DCN) ${ }^{9}$ para os cursos de Odontologia, publicadas em 2002, exigiram formação integral, humana, cidadã e transformadora da realidade, incluindo mudanças no processo de ensino-aprendizagem que possibilitassem o domínio de seis competências gerais no estudante: Atenção à saúde, Comunicação, Tomada de Decisão, Educação Permanente, Liderança e Administração e Gerenciamento. O currículo proposto pelas DCN demanda um perfil de egresso com domínios cognitivos, afetivos e psicomotores nas áreas de formação geral, formação profissional e cidadania.

Ao considerar a atitude como uma tarefa de aprendizagem, não se pode negligenciar os dez aspectos relacionados ao fenômeno denominado ecologia da aprendizagem: o próprio aprendiz, o contexto educacional, o currículo, os sistemas de avaliação da aprendizagem e institucional, as metodologias de ensino e materiais didáticos, o docente e a organização da instituição educacional $^{12}$.

Sendo assim, a literatura destaca a importância da avaliação de atitudes em situações específicas $^{13-16}$, principalmente inserida como componente em avaliações das práticas ${ }^{17}$.
Pesquisadores brasileiros ${ }^{18-20}$ da área de educação médica desenvolveram instrumentos, com o objetivo de aferir aspectos relevantes ao exercício da profissão e conceitos apresentados nas DCN, bem como ressaltaram a relevância da aplicação de tais ferramentas a fim de fundamentar discussões sobre currículos ${ }^{21}$.

Outros instrumentos baseados em escalas de auto relato foram desenvolvidos e, posteriormente, validados na língua portuguesa para medir características dos estudantes no processo de formação em saúde que remetem ao perfil de egresso contemporâneo. É o caso da validação dos instrumentos que medem empatia na relação profissional-paciente ${ }^{22}$ e aprendizagem ao longo da vida ${ }^{15}$, esta última, propõe avaliar o compromisso do estudante com sua formação continuada e prática baseada em evidências. Há, ainda, as medidas de orientação para aprendizagem colaborativa interprofissional ${ }^{23,24}$.

Apesar das publicações em educação para profissionais de saúde, atitudes e comportamentos relacionados às competências gerais de formação costumam ser negligenciados pelos sistemas de avaliação do ensino superior do Brasil, onde são avaliados desempenhos dos estudantes por meio de teste cognitivo, condições da oferta do curso, bem como, por avaliações institucionais internas e externas $^{25}$.

Quando se analisa os cursos de saúde, não se observa a análise das atitudes numa visão global das competências curriculares, sob a perspectiva de avaliação formativa ${ }^{14}$ e como recurso de autoavaliação institucional em Odontologia, apesar desse construto ser essencial. Dessa forma, identifica-se demanda por um instrumento que permita, por meio de uma autoavaliação de comportamentos, mensurar o grau de desenvolvimento das habilidades atitudinais e, consequentemente, guiar a construção de conhecimentos para o alcance das competências desejadas para a formação profissional, auxiliando 
na discussão de currículos de Odontologia.

Diante dessa premissa, o objetivo do presente estudo é descrever o processo de elaboração da matriz de indicadores e dos itens desenvolvidos para compor uma escala de atitudes relacionadas às competências odontológicas.

\section{METODOLOGIA}

Tratou-se de um estudo de desenvolvimento metodológico $^{26}$, para construção e validação de conteúdo e de face de uma escala que ocorreu com um grupo de especialistas e com uma amostra de acadêmicos (piloto). Integrou um estudo de doutorado conduzido pela primeira autora do presente artigo.

Após aprovação do projeto pelo Comitê de Ética em Pesquisa (CAAE 50727215.6. 0000.5208), deu-se início aos procedimentos metodológicos de validação da escala, envolvendo sete passos (figura 1).

A metodologia para sistematização do modelo teórico ${ }^{27}$ culminou na estruturação de uma matriz com seis indicadores, estabelecidos com base na literatura de educação para profissionais de saúde e documentos orientadores da profissão de cirurgião-dentista (quadro 1).

A metodologia empregada para o consenso dos especialistas foi a Técnica do Grupo Nominal $(\mathrm{TGN})^{27,28}$, com participação de cirurgiõesdentistas com, no mínimo, dez anos de experiência, atuantes nas áreas de gestão $(n=3$, na área de educação), assistência ( $n=3$, atuando em atenção primária, secundária ou terciária, com experiência em preceptoria) ou docência $(n=4)$, que frequentaram, pelo menos uma vez, a Reunião Anual da Associação Brasileira de Ensino Odontológico (ABENO) nos últimos cinco anos.

Após o contato inicial, a TGN foi conduzida em dois momentos: julgamento virtual realizado de forma independente, assíncrona e virtual (Tempo 1 - T1). Após 15 dias (Tempo 2 - T2), discussão presencial com o objetivo de convergir as respostas, chegando a um consenso.

A análise dos indicadores teve abordagem qualitativa (de acordo com sua relevância e importância como "indispensável", "necessário" ou "dispensável") e quantitativa (pontuação de 0 a 6: 0 = "sem importância", 1 = "quase sem importância", 2 = "pouco importante", 3 = "importância media", 4 = "importante", 5 = "muito importante"; e $6=$ "o mais importante") ${ }^{27}$, bem como requeria o preenchimento de uma matriz em que o especialista correlacionou o indicador com a(s) competência(s) odontológica(s).

Em T2 houve momento de consenso presencial e resposta individual. O indicador foi excluído quando em $\mathrm{T} 2$ recebeu classificação de “dispensável”, pela análise qualitativa ou obteve, na sua classificação quantitativa, um percentual igual ou superior a 50\% na nota máxima atribuída (6), entre os membros do grupo ${ }^{27}$. Elaborou-se uma síntese da discussão ocorrida em T2 que foi enviada ao grupo de especialistas para ratificação de seu conteúdo.
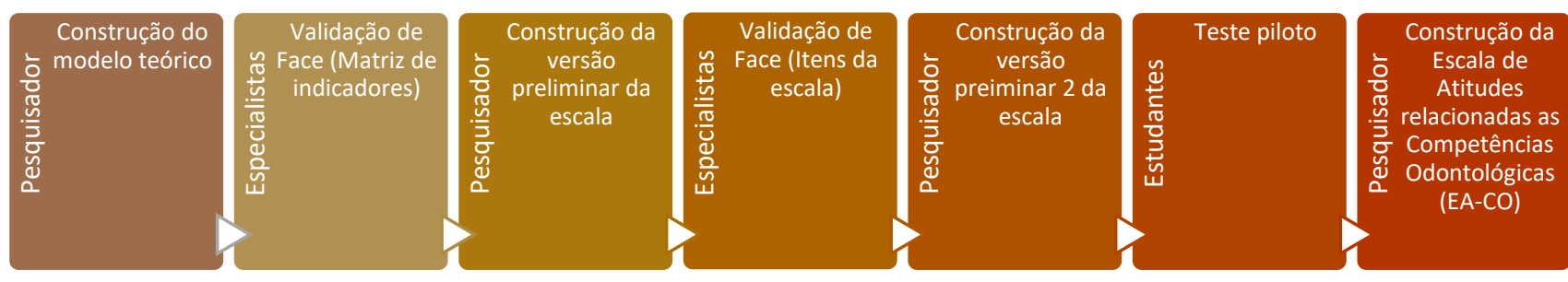

Figura 1. Etapas metodológicos da elaboração e validação da escala 
Quadro 1. Modelo teórico e matriz de indicadores da escala de atitudes relacionadas às competências odontológicas (EA-CO)

\begin{tabular}{|c|c|c|c|c|c|c|c|c|}
\hline \multirow{2}{*}{ Indicador } & \multirow{2}{*}{ Conceito adotado no Modelo Teórico } & \multirow{2}{*}{$\begin{array}{l}\text { Autores } \\
\text { (Ano) }\end{array}$} & \multicolumn{6}{|c|}{$\begin{array}{l}\text { Competências } \\
\text { Odontológicas DCN* }\end{array}$} \\
\hline & & & 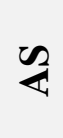 & 를 & $\sum^{\sum}$ & 寻 & 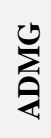 & A \\
\hline $\begin{array}{l}\text { Empatia na } \\
\text { relação } \\
\text { profissional- } \\
\text { paciente }\end{array}$ & $\begin{array}{l}\text { Considerando empatia como um atributo predominantemente cognitivo (se opondo ao } \\
\text { afetivo ou emocional) que envolve um entendimento (se opondo ao sentimento) da dor, } \\
\text { experiências, queixas e perspectivas dos pacientes, combinada com a capacidade de } \\
\text { comunicar esse entendimento e na intenção de ajudá-lo, através da prevenção e alívio } \\
\text { da dor e sofrimento. }\end{array}$ & $\begin{array}{l}\text { Hojat et al. } \\
(2002)^{13} \text {; } \\
\text { Hojat e } \\
\text { Lanoue } \\
(2014)^{43}\end{array}$ & $\mathrm{X}$ & $\mathrm{X}$ & $\mathrm{X}$ & $\mathrm{X}$ & & \\
\hline $\begin{array}{l}\text { Aprendizagem ao } \\
\text { longo da vida }\end{array}$ & $\begin{array}{c}\text { Motivação, capacidade e comportamentos necessários à manutenção e ao } \\
\text { desenvolvimento da competência ao nível dos conhecimentos, das tecnologias e das } \\
\text { práticas mais recentes }\end{array}$ & $\begin{array}{l}\text { Salgueira et } \\
\text { al. }(2009)^{15} \text {. }\end{array}$ & $\mathrm{X}$ & $\mathrm{X}$ & $X$ & $\mathrm{X}$ & $\mathrm{X}$ & $\mathrm{X}$ \\
\hline $\begin{array}{l}\text { Disponibilidade } \\
\text { para } \\
\text { aprendizagem } \\
\text { interprofissional }\end{array}$ & $\begin{array}{l}\text { Ações positivas em uma proposta onde duas ou mais profissões aprendem juntas sobre } \\
\text { o trabalho conjunto e sobre as especificidades de cada uma, na melhoria da qualidade } \\
\text { no cuidado ao paciente }\end{array}$ & $\begin{array}{l}\text { Williams et al. } \\
\quad(2012)^{16} \\
\text { Aguilar da } \\
\text { Silva et al. } \\
(2011)^{23}\end{array}$ & $\mathrm{X}$ & $\mathrm{X}$ & $X$ & $X$ & $\mathrm{X}$ & $\mathrm{X}$ \\
\hline $\begin{array}{l}\text { Proteção aos } \\
\text { direitos dos } \\
\text { pacientes }\end{array}$ & $\begin{array}{l}\text { Aspectos éticos sobre o comportamento profissional durante a informação ao paciente e } \\
\text { sua autonomia na decisão do plano de tratamento. }\end{array}$ & $\begin{array}{l}\text { Chiu et al. } \\
(2010) ;{ }^{44} \text { CFO } \\
(2012)^{45}\end{array}$ & $\mathrm{X}$ & $\mathrm{X}$ & $\mathrm{X}$ & $\mathrm{X}$ & & \\
\hline $\begin{array}{l}\text { Responsabilidade } \\
\text { Social (RS): }\end{array}$ & $\begin{array}{c}\text { Garantia de atendimento em todos os níveis de complexidade, interação com a } \\
\text { comunidade, utilização racional de recursos diagnósticos e terapêuticos, e prevenção de } \\
\text { doenças }\end{array}$ & $\begin{array}{l}\text { Miranda et al. } \\
\quad(2009)^{20}\end{array}$ & $\mathrm{X}$ & & & $\mathrm{X}$ & $\mathrm{X}$ & $\mathrm{X}$ \\
\hline $\begin{array}{c}\text { Inteligência } \\
\text { Emocional (IE): }\end{array}$ & Envolve a percepção, processamento, regulação e manejo das emoções. & $\begin{array}{l}\text { Arora et al. } \\
(2010)^{52}\end{array}$ & $\mathrm{X}$ & & $\mathrm{X}$ & $\mathrm{X}$ & & \\
\hline
\end{tabular}

AS: Atenção à Saúde; TD: Tomada de Decisões; COM: Comunicação; LID: Liderança; ADMG: Administração e Gerenciamento; EP: Educação Permanente 
O mesmo referencial teórico serviu de fonte para elaboração dos itens pelo pesquisador, compondo o passo metodológico 3. Cada indicador originou entre 10 e 11 itens, conforme os conceitos estabelecidos no modelo teórico validado pelos especialistas, resultando na versão preliminar 1 com 53 itens. Procurou-se delinear itens de fácil entendimento, objetivos e relevantes ${ }^{29,30}$, assim como que representassem comportamentalmente atitudes relacionadas às competências (traços latentes). A garantia do caráter comportamental foi estabelecida por um profissional da área de psicologia.

No passo 4, ocorreu a validação de face dos itens da escala analisados. A metodologia empregada foi aplicação de questionário online na plataforma SurveyMonkey® com julgamento qualitativo quanto à relevância no modelo teórico (“indispensável”, “necessário” ou “dispensável”). Foi oferecido espaço para comentários com sugestões de nova redação, corroborando para uma melhor compreensão ou inclusão de um novo item. Os especialistas classificaram a abrangência sobre o construto e seus domínios. O julgamento foi qualitativo e em tempo único, de modo que para exclusão adotou-se um ponto de corte, quando o item teve acima de $50 \%$ de reprovação (categoria “dispensável”).

Após os ajustes indicados pelos especialistas, o pesquisador elaborou a versão preliminar $2 \mathrm{da}$ escala com cinco opções de resposta tipo Likert: "discordo totalmente" (1), "discordo parcialmente" (2), "Nem concordo, nem discordo" (3), "concordo parcialmente" (4) e "concordo totalmente" (5) (passo 5) que foi aplicada a uma amostra piloto de estudantes, integrantes da população-alvo do presente estudo (passo 6). Foram convidados 30 alunos de uma das instituições de ensino superior (IES) participantes para um encontro prévio a um evento organizado pela instituição, solicitando o comparecimento com quatro horas de antecedência. Buscou-se representatividade de todos os períodos e ambos os gêneros.

No passo 6, os estudantes responderam a escala e o questionário com informações sociodemográficas da pesquisa, com registro do tempo de resposta e, em seguida, aplicou-se um questionário para analisar a versão preliminar 2 da escala quanto à clareza, formatação e orientações de preenchimento, número de questões e compreensão dos termos, bem como a abrangência do objetivo e fidelidade das respostas. A discussão desses aspectos ocorreu coletivamente, explorando as soluções para maior clareza e compreensão, a fim de garantir a qualidade das respostas em todos os estágios da formação odontológica. $\mathrm{O}$ passo 7 foi a edição da versão final da escala de atitudes relacionadas às competências odontológicas.

\section{RESULTADOS \\ Validação de face da matriz de indicadores}

Observou-se que o indicador da Inteligência Emocional recebeu percentual abaixo do ponto de corte estabelecido, tanto na análise qualitativa (relevância) (gráfico 1) quanto na quantitativa (grau de importância) (gráfico 2), corroborando para sua exclusão do modelo, após a TGN.

Discutiu-se a dificuldade de relacionar os indicadores com apenas uma competência, uma vez que as mesmas não se isolam e, sim, se complementam. Tanto os indicadores, quanto as competências entre si apresentam uma correlação importante, sendo quase impossível fragmentá-las.

$\mathrm{O}$ indicador da Inteligência Emocional (IE) apresentou divergência no processo de avaliação. Quanto ao conceito: inicialmente, foi questionado por um dos especialistas, no tocante ao proposto no modelo, que não estaria claro o suficiente. Quanto à importância: muitas falas mostraram a importância da IE na vida profissional: na resolução de problemas, para lidar com pacientes terminais, com a morte de pacientes, para trabalhar em equipes multiprofissionais e na atenção primária. Quanto à pertinência no modelo 
proposto: foi questionado acerca do tamanho do atitude deve ser incluída na avaliação, de modo que instrumento necessário para mensurar esse esse indicador poderia ser parcialmente construto e também sobre as prioridades de qual interpretado por outros já existentes no modelo.

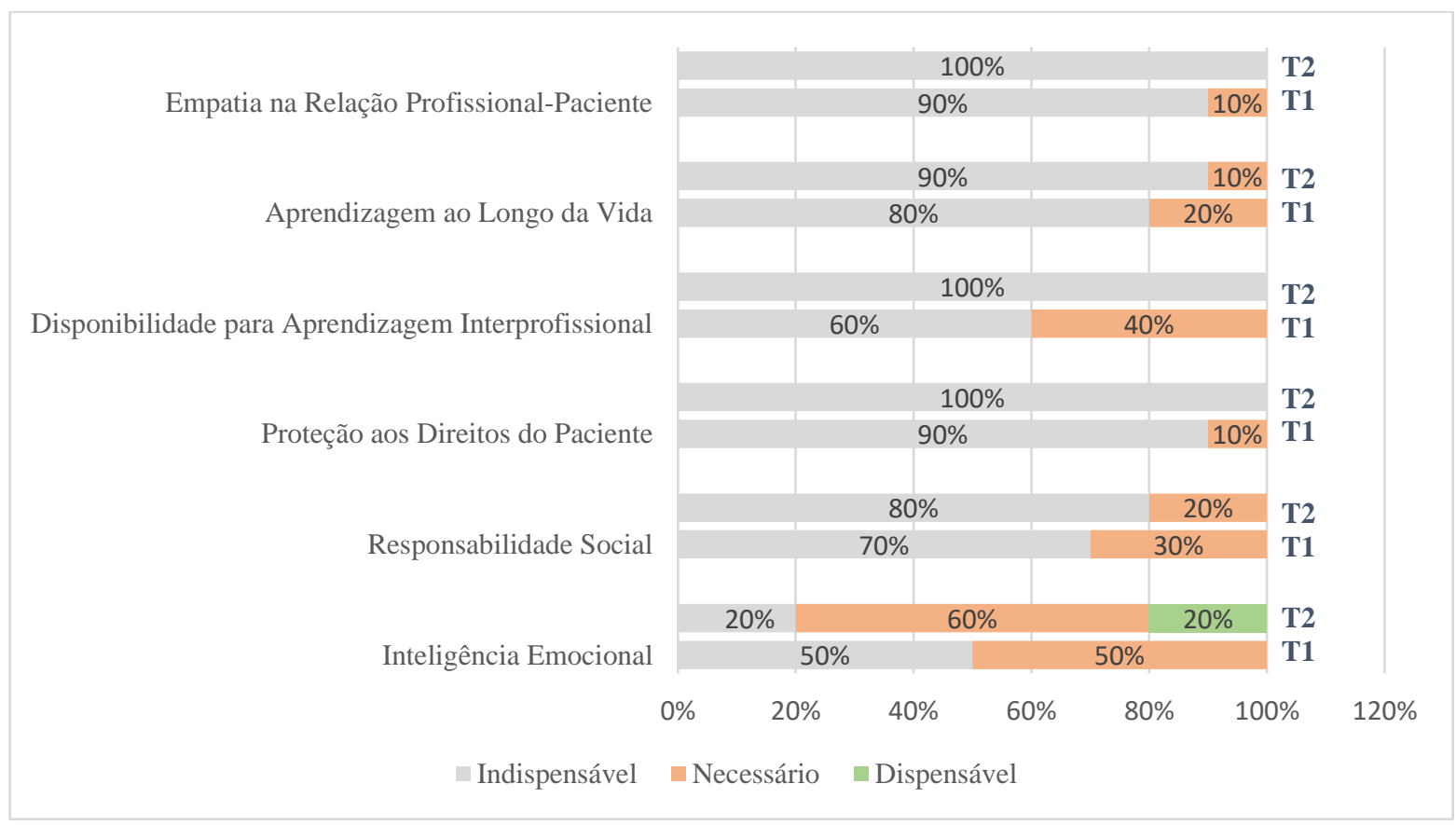

Gráfico 1. Distribuição das respostas da análise qualitativa dos especialistas antes (T1) e após (T2) a discussão presencial

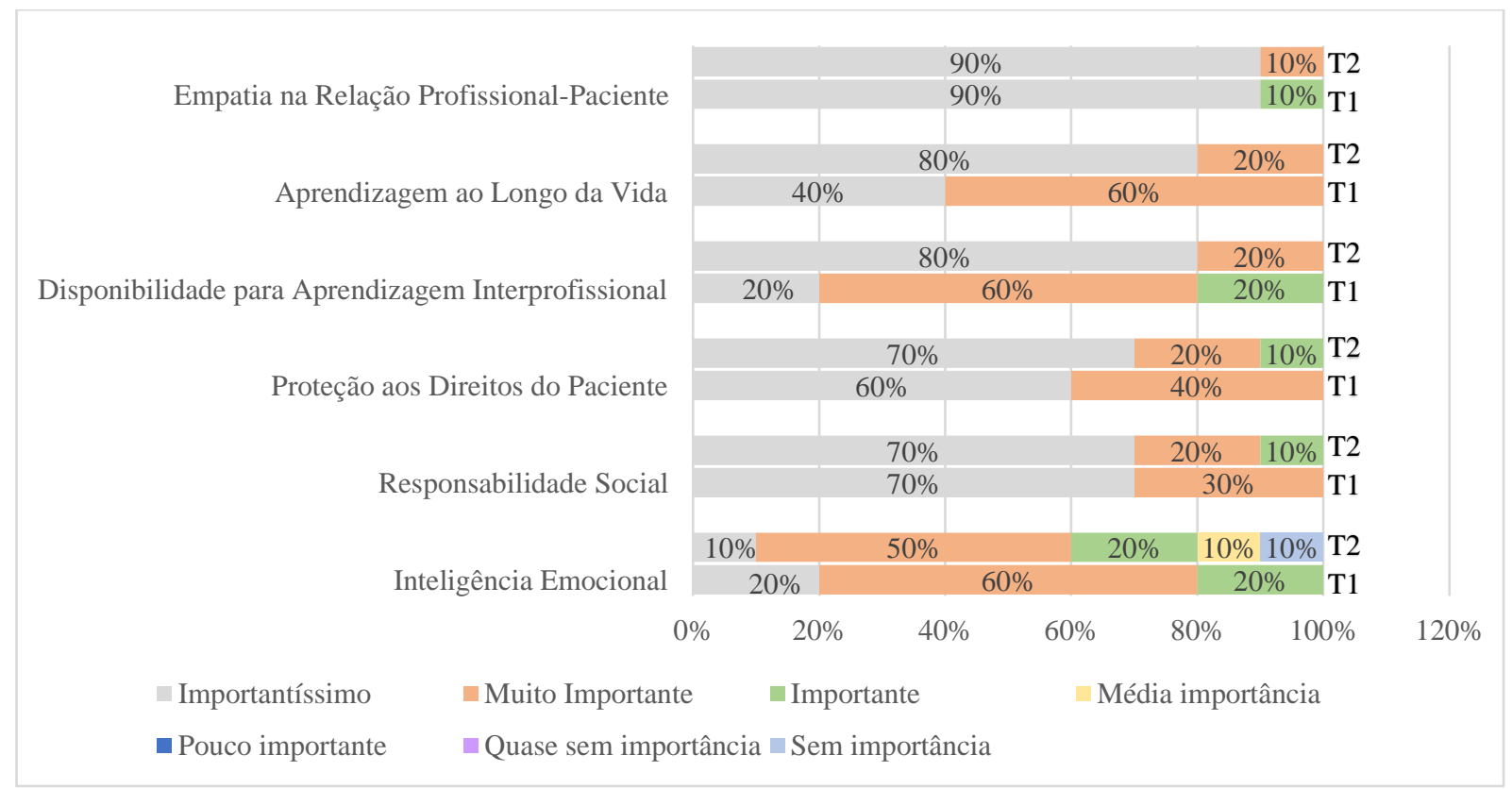

Gráfico 2. Distribuição das respostas da análise quantitativa dos especialistas antes (T1) e após (T2) a discussão presencial 
Verificou-se que houve melhora no consenso para os demais indicadores. Estes foram relacionados com as competências de modo representativo, considerando as médias das proporções atribuídas aos indicadores em T1 e T2 (gráfico 3).

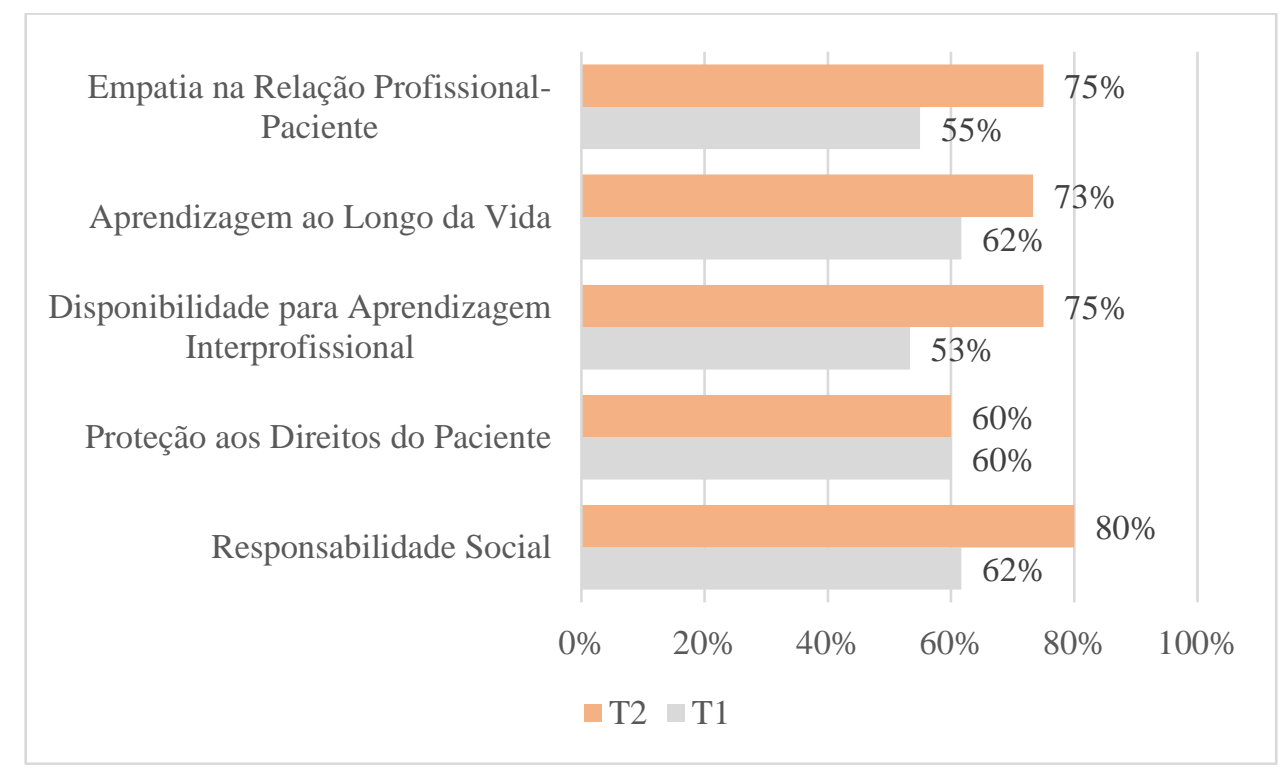

Gráfico 3. Média das proporções estabelecidas na relação indicadores X competências odontológicas antes (T1) e após (T2) a discussão presencial

Um dos especialistas se posicionou favoravelmente, uma vez que "por sermos seres humanos, esse indicador deve ser avaliado nos estudantes", pois "todos nós somos razão e emoção e saber lidar com as emoções é essencial para uma formação integral e humana."

Os argumentos contrários à inclusão da IE no modelo foram: "incerteza da possibilidade de mensurar e trabalhar cognitivamente na docência" (gestor educacional), "dificuldade de ensinar e de influenciar os estudantes" (docente), "falta de estabilidade" (gestor), "por ser fruto de amadurecimento pessoal com pouca influência da docência e ser suscetível a mudanças durante a formação e estado de saúde mental" (docente) e pela "possibilidade de ser influenciado por genética” (docente).

\section{Validação de face dos itens da escala}

Os especialistas julgaram relevante a composição da versão preliminar 1 da escala, de modo que $100 \%$ a consideraram abrangente em relação ao construto. Sugeriram, no entanto, modificações na redação de nove dos 53 itens.

\section{Estudo piloto com a população-alvo da escala}

A amostra piloto foi composta de 17 dos 30 estudantes convidados, distribuídos conforme estágio de formação em: Inicial $\left(1^{\circ}\right.$ ao $3^{\circ}$ período) (41,2\%); Médio ( $4^{\circ}$ ao $6^{\circ}$ período) $(29,4 \%$ ); e Final ( $7^{\circ}$ aos $10^{\circ}$ períodos $)(29,4 \%)$. A faixa etária situou-se entre 19 a 27 anos de idade, a maioria do turno diurno (n-12/70,6\%) e homens $(\mathrm{n}=9 / 52,9 \%)$. Relataram satisfação quanto à formatação do instrumento $(88,2 \%)$, clareza e objetividade dos itens $(94,1 \%)$, ao objetivo da escala $(100 \%)$ e ao número de questões $(58,8 \%)$, assegurando fidelidade nas suas respostas (70,6\%). Destaca-se que $58,8 \%$ dos estudantes encontraram pelo menos 1 item com termos desconhecidos, adaptados posteriormente.

No quesito de fidelidade das respostas, a 
maioria conseguiu responder ao instrumento "pensando/sentindo quais eram ou seriam suas atitudes nas situações apresentadas", sendo que $29,4 \%$ dos estudantes declararam que "suas respostas correspondiam às atitudes mais aceitas pela sociedade, representando também as suas atitudes".

A fim de solucionar a incompreensão dos termos, houve edição de quatro itens que após alterações geraram a versão final da escala de atitudes relacionadas às competências odontológicas (quadro 2).

O tempo para resposta ao questionário da pesquisa com dados sociodemográficos e educacionais e à escala variou de 5 minutos e 10 segundos a 11 minutos e 30 segundos, com média de aproximadamente 8 minutos.

\section{DISCUSSÃO}

A proposta de criação de uma escala para avaliação de atitudes relacionadas às competências odontológicas atende a uma demanda na área do ensino de Odontologia e de assistência à saúde no SUS, uma vez que existe excesso de profissionais formados sob a perspectiva tecnicista, enquanto as habilidades atitudinais são negligenciadas na prática assistencial $^{31}$, curricular $^{32}$ e em sistemas de avaliação governamentais ${ }^{25}$ e institucionais ${ }^{14,32}$.

Não obstante, publicações coordenadas por representantes da Associação Brasileira de Ensino Odontológico ${ }^{33,34}$ realçaram a dificuldade das instituições brasileiras em adotar a proposta curricular das DCN que apresentavam inovações tanto nos aspectos pedagógicos, quanto na composição das competências gerais em áreas não técnicas da profissão. Isso pode ser exemplificado no estudo ${ }^{32}$ que ocorreu a análise da dimensão ética nos cursos de Odontologia, em que se observou a inexpressividade de tal dimensão em vivências tutoriais e de avaliação.

$\mathrm{O}$ presente estudo pretendeu contribuir para uma melhora no cenário descrito e atentou para evidências de validade de uma escala de atitudes relacionadas às competências odontológicas. Para tal, foram considerados parâmetros nacionais ${ }^{26,35}$ e internacionais ${ }^{36,37}$ nas etapas metodológicas.

Para que um instrumento apresente uma boa qualidade, durante a fase de validação de conteúdo, é necessário apresentar claramente seu objetivo; a população alvo a que se destina; adequada definição e fundamentação teórica dos conceitos, embasados em literatura pertinente; além de mencionar como ocorreu a seleção de itens, de forma que facilite a leitura $\mathrm{e}$ compreensão do instrumento, envolvendo a população alvo e os experts no assunto ${ }^{36}$. Todos esses aspectos foram envolvidos nos passos da metodologia do presente estudo.

Destaca-se que, quanto ao objetivo da escala, a mesma tem caráter avaliativo e formativo, pois a intenção é de que professores e estudantes atentem para as questões das atitudes relacionadas às competências, desde o início da formação até os estágios finais da formação. As medidas de autorrelato sobre atitudes no campo educacional só têm efetividade quando funcionam como avaliação formativa ${ }^{2}$. Esta, por sua vez, propicia o desenvolvimento das habilidades individualizada e com graus de complexidade crescente, devendo vir acompanhadas por devolutivas (feedback) ${ }^{38-40}$.

Outra possibilidade, seria utilizar os dados originados pela escala com o objetivo de avaliação de práticas curriculares, como ocorreu com o instrumento desenvolvido previamente ${ }^{20}$ que pretendeu avaliar atitudes de estudantes de Medicina, sendo aplicados de forma longitudinal no estudo posterior ${ }^{21}$ apontando fortalezas e fragilidades tanto individuais quanto coletivas.

Quanto ao construto do instrumento, cabe uma breve discussão sobre o conceito de atitude incluído no modelo. Embora tenha sido utilizado 
Quadro 2. Versão final dos itens para o instrumento de avaliação de atitudes relacionadas às competências odontológicas após validação de face com especialistas e amostra piloto da população-alvo

\begin{tabular}{|c|c|c|c|c|c|}
\hline & $\begin{array}{c}\text { Empatia na Relação } \\
\text { Profissional- } \\
\text { Paciente }^{13,20,22,43,58,59,60} \\
\end{array}$ & $\begin{array}{l}\text { Aprendizagem ao Longo da } \\
\text { Vida }^{15,20,48}\end{array}$ & $\begin{array}{l}\text { Disponibilidade para Aprendizagem } \\
\text { Interprofissional }{ }^{16,20,23,24,49,61}\end{array}$ & Responsabilidade Social $^{20,31}$ & $\begin{array}{l}\text { Proteção aos Direitos do } \\
\text { Paciente }^{44,45}\end{array}$ \\
\hline 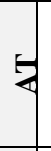 & $\begin{array}{l}\text { Adoção de perspectiva; Cuidar com } \\
\text { humanidade; Comunicação e } \\
\text { interação }\end{array}$ & $\begin{array}{l}\text { Motivação e crenças relacionadas } \\
\text { com a aprendizagem; Atenção a } \\
\text { oportunidades de aprendizagem; } \\
\text { Autoaprendizagem }\end{array}$ & $\begin{array}{l}\text { Trabalho em equipe e colaboração; Trabalho } \\
\text { em equipe; Aprendizagem cooperativa }\end{array}$ & $\begin{array}{l}\text { Resolutividade; Educação em saúde } \\
\text { Intersetorialidade; Consciência social e } \\
\text { sanitária }\end{array}$ & $\begin{array}{l}\text { Informação do paciente; } \text { Autonomia } \\
\text { do paciente nas decisões } \\
\text { terapêuticas }\end{array}$ \\
\hline & $\begin{array}{l}\text { 1. Eu percebo quando o meu paciente } \\
\text { está com medo/ansioso durante a } \\
\text { consulta } \\
\text { 2. Acredito que percebendo os } \\
\text { sentimentos dos meus pacientes, } \\
\text { tenho mais chances de obter melhores } \\
\text { resultados terapêuticos } \\
\text { 3. Ouço as queixas do paciente com } \\
\text { atenção com o objetivo de elaborar } \\
\text { um planejamento individualizado } \\
\text { 4. Utilizo expressões não verbais, } \\
\text { como o aperto de mão e balançar a } \\
\text { cabeça afirmativamente, durante a } \\
\text { consulta, para melhorar a } \\
\text { comunicação com o paciente } \\
\text { 5. Eu entendo o que o paciente sente } \\
\text { com sua doença, sem sentir a sua dor } \\
\text { 6. A minha postura diante do paciente } \\
\text { não depende da sua aparência (física } \\
\text { ou condição social) } \\
\text { 7. Durante consulta de anamnese } \\
\text { (história da doença) do paciente, } \\
\text { escuto mais do que falo } \\
\text { 8. Fico atento ao tom da minha voz } \\
\text { durante a anamnese e nas demais } \\
\text { consultas } \\
\text { 9. Demonstro ao paciente que } \\
\text { compreendo suas queixas } \\
\text { 10. Cumprimento o paciente no início } \\
\text { da consulta }\end{array}$ & $\begin{array}{l}\text { 11. Quando vejo um assunto } \\
\text { desconhecido e interessante, procuro } \\
\text { me informar mais sobre ele } \\
\text { 12. Mesmo que não goste da } \\
\text { disciplina, estudo para ser um bom } \\
\text { profissional } \\
\text { 13. A faculdade me motiva a estudar } \\
\text { para ser um bom profissional } \\
\text { 14. Sei avaliar o meu desempenho } \\
\text { numa atividade acadêmica } \\
\text { 15. As avaliações da faculdade } \\
\text { contribuem para que eu aprenda com } \\
\text { os erros } \\
\text { 16. Participar de pesquisa científica, } \\
\text { mesmo sem bolsa, me motiva } \\
\text { 17. Considero as atividades de } \\
\text { extensão uma oportunidade de } \\
\text { aprendizagem } \\
\text { 18. } \\
\text { reuniões/encontros/congressos na } \\
\text { área de odontologia é estimulante } \\
\text { 19. Pretendo continuar estudando } \\
\text { quando já estiver formado } \\
\text { 20. Utilizo fontes de informações } \\
\text { variadas (resumos, livros, artigos } \\
\text { científicos, internet) para estudar } \\
\text { 21. Acredito que após } 20 \text { anos de } \\
\text { formado, ainda estarei estudando } \\
\text { temas de odontologia para me } \\
\text { atualizar }\end{array}$ & $\begin{array}{l}\text { 22. Estudar com alunos de outros cursos na } \\
\text { graduação melhora minha prática odontológica } \\
\text { 23. Respeito os saberes de alunos de outras } \\
\text { graduações } \\
\text { 24. Penso que as diversas áreas da saúde } \\
\text { contribuem para melhorar a saúde de um } \\
\text { doente, de forma igual } \\
\text { 25. O trabalho em equipe multi/interprofissional } \\
\text { me permite aprender a me relacionar com as } \\
\text { pessoas equipes } \\
\text { 26. A experiência em } \\
\text { multi/interprofissionais é fundamental para } \\
\text { minha formação de cirurgião-dentista } \\
\text { 27. Para discutir um caso clínico com } \\
\text { profissionais de outras áreas, preciso estudar } \\
\text { mais } \\
\text { 28. Buscando a resolução de um problema, } \\
\text { trabalho melhor em um grupo pequeno de } \\
\text { estudantes do que num grande grupo } \\
\text { 29. Acredito que o trabalho colaborativo em } \\
\text { grupos multi/interprofissionais tem mais chance } \\
\text { de obter resultados positivos para o paciente } \\
\text { 30. Compartilhar experiências com estudantes } \\
\text { de outros cursos de saúde me torna uma pessoa } \\
\text { melhor } \\
\text { 31. Minha comunicação interprofissional } \\
\text { melhora se na graduação eu tiver experiência } \\
\text { acadêmica com alunos de outros cursos } \\
\text { 32. Considero que a liderança de uma equipe } \\
\text { multi/interprofissional pode ser ocupada por } \\
\text { qualquer área profissional de saúde }\end{array}$ & 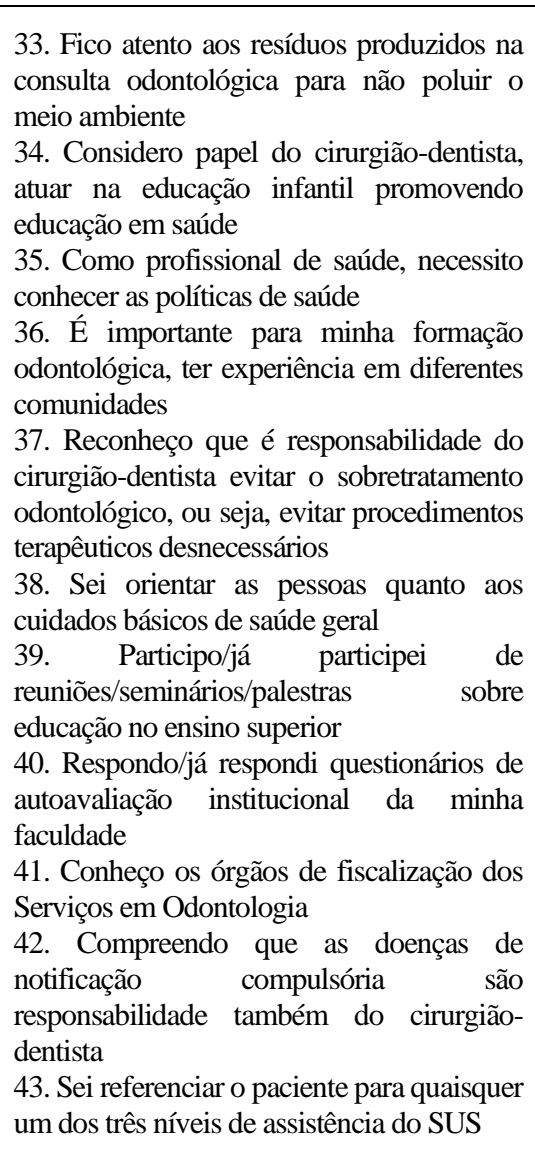 & $\begin{array}{l}\text { 44. Acho válido existirem } \\
\text { associações que protejam os direitos } \\
\text { dos usuários de clínicas de Ensino } \\
\text { Odontológico } \\
\text { 45. Compreendo que a alta do } \\
\text { tratamento odontológico deve ser } \\
\text { pactuada com os pacientes } \\
\text { 46. Permito que o meu paciente } \\
\text { participe da decisão do tratamento } \\
\text { 47. Meu paciente tem o direito de } \\
\text { conhecer mais de uma opção } \\
\text { terapêutica para a sua doença } \\
\text { 48. Não publico fotos dos meus } \\
\text { pacientes sem seu consentimento por } \\
\text { escrito } \\
\text { 49. Se meu paciente quiser, forneço a } \\
\text { documentação odontológica para ele } \\
\text { e faço cópia para arquivar } \\
\text { 50. Só inicio o tratamento } \\
\text { odontológico de rotina, quando meu } \\
\text { paciente assina termo de } \\
\text { consentimento } \\
\text { 51. Considero que pacientes com } \\
\text { doenças graves precisam saber do seu } \\
\text { estado, desde que desejem sabê-lo } \\
52 \text {. Ao atender uma criança, } \\
\text { direciono as informações pertinentes } \\
\text { para ela e para seu responsável } \\
53 \text {. Ao participar de pesquisa } \\
\text { científica, realizo a coleta de dados } \\
\text { somente após assinatura do termo de } \\
\text { consentimento pelo paciente }\end{array}$ \\
\hline
\end{tabular}


um conceito da psicologia social ${ }^{1}$, o mesmo é coerente com o conceito no campo educacional, sob a perspectiva de funcionar como elementos essenciais para o desenvolvimento de competências $^{2,7,14}$. A articulação em rede das competências gerais das DCN é citada na literatura ${ }^{41,34}$ e fica evidente quando se buscou indicadores que indiretamente pudessem representá-las. Os resultados na validação de face da matriz de indicadores retratam tal indissociabilidade das competências, quando é afirmada pelo grupo de especialistas a dificuldade de relacioná-las com apenas uma das competências.

Quantos aos indicadores da escala, o termo empatia aparece nas DCN na descrição do conceito da competência da Liderança, certamente considerando sua importância no processo de comunicação com os pares. A proposta do modelo desse estudo ressaltou a empatia na relação profissional-paciente por contemplar a competência da Atenção à Saúde e a da Comunicação. No ensino da comunicação profissional-paciente, a empatia é citada como elemento-chave para construção de uma relação que pode ser ensinada durante a formação do profissional de saúde ${ }^{42}$. Indiretamente, sabendo respeitar e valorizar o paciente, a relação com pares e em situação de liderança estaria garantida. Inúmeros instrumentos tratam dessa relação, apresentando evidências de validade ${ }^{13,43}$.

A Proteção aos Direitos do Paciente foi incluída na proposta enfatizando a informação e autonomia do paciente nas decisões terapêuticas ${ }^{44}$ e por documentos que normatizam a profissão do odontólogo brasileiro ${ }^{45}$. A ênfase na relação com o paciente é observada com a inclusão de indicadores que proveem a segurança do paciente na relação com profissional de saúde. A relação é majoritariamente paternalista, e um instrumento que avalia atitudes deve desencorajar essa cultura tanto na formação quanto na prática profissional. Na literatura, as avaliações da competência clínica são acrescidas desses componentes, e tem seu valor reconhecido ${ }^{46,47}$.

A inclusão do indicador Aprendizagem ao Longo da Vida (ALV) tem evidência na literatura de formação de profissionais de saúde, de modo que há instrumento próprio validado na língua portuguesa que retrata a combinação de profissionalismo, motivação e crenças relacionadas com a aprendizagem, atividades acadêmicas, atenção a oportunidades de aprendizagem e competências técnicas de procura de informação para médicos ${ }^{15,48}$. Nas DCN, essa necessidade foi evidenciada na descrição da competência geral da Educação Permanente, Tomada de Decisões e indiretamente na Atenção à Saúde, uma vez que o padrão de qualidade da assistência prestada envolve a atualização constante e acesso à informação.

Foi questionada pelo grupo a possibilidade de superestimar a relevância da ALV no modelo, por se tratar de um grupo de especialistas com alto nível de formação, no entanto é esperado que o egresso seja "cirurgião-dentista generalista, dotado de sólida formação técnico-científica e ativo na construção permanente de seu conhecimento" 34,41 .

O indicador Disponibilidade para Aprendizagem Interprofissional abordou a realidade da atuação em equipes multiprofissionais, considerada como garantia de qualidade do atendimento à saúde do indivíduo e coletividade desde a implementação do Sistema Único de Saúde. O termo interprofissional foi preferido para que essa atuação de forma integrada entre as diferentes profissões ocorra concomitantemente, pretendendo alcançar a prática colaborativa e melhores resultados 
terapêuticos ${ }^{49}$. Detectar a disponibilidade para a aprendizagem interprofissional no graduando, como proposto no modelo, propicia feedbacks relacionados ao tema que na Odontologia é extremamente restrito ${ }^{50}$. Esse indicador coaduna com diretrizes para práticas interprofissionais de extensão, desafio para os currículos de graduação ${ }^{51}$.

Complementando a análise dos indicadores estabelecidos e as DCN, a palavra responsabilidade aparece na descrição de três das seis competências gerais das DCN: Atenção à Saúde, Liderança e Educação Permanente. Não obstante, a Responsabilidade Social (RS) é citada no instrumento de avaliação de atitudes criado para estudantes de Medicina ${ }^{20}$ que fundamentou o conceito presente no modelo, corroborando com a realidade das $\mathrm{DCNs}^{9}$ e das diretrizes de extensão ${ }^{51}$.

A exclusão da Inteligência Emocional no modelo se deu mais por questões de ordem técnica, haja vista que o conceito não foi desvalorizado pelo grupo de especialistas, como evidenciado na seção de resultados, contudo foi considerado bastante amplo, o que por si só demandaria uma gama de dimensões ${ }^{52,53}$, inviabilizando sua inclusão no modelo.

Além da recomendação de incluir objetivo e adequada definição do construto e seus domínios no processo de construção de instrumentos, a literatura orienta que durante a validação de face dos itens da escala, devem ser observados três aspectos: a abrangência dos itens sobre aspectos relevantes do conteúdo, bem como a de seus domínios, e a fundamentação teórica para a descrição clara do construto e sua teoria de referência ${ }^{37}$. Os autores do presente estudo atenderam a esse critério, seguindo passos metodológicos de forma criteriosa, como descrita na seção específica desse artigo. Exemplifica-se os resultados para o estabelecimento de uma quantidade uniforme de itens por indicador
(Gráfico 3), aliada a orientação de atender a abrangência teórica dos conceitos satisfatoriamente ${ }^{35}$.

Já com a participação da população-alvo no processo de validação por meio de estudo piloto, é possível dimensionar o grau de compreensão dos itens da escala. $\mathrm{O}$ relato da fidedignidade de suas respostas, bem como a discussão sobre os indicadores do modelo teórico, aumentou as evidências de validade quanto à abrangência e formatação dos itens. A percepção de acadêmicos de Odontologia na análise curricular e competências adquiridas sob a ótica das DCN já foi descrita na literatura com 91 alunos do último ano do curso de uma IES no sul do Brasil $^{54}$. Os autores encontraram, após reforma curricular, resultados positivos em ordem decrescentes para: 1) o desenvolvimento de ações de prevenção, promoção, proteção e reabilitação da saúde; 2) a disposição ao aprendizado contínuo; 3) a tomada de decisões; 4) e a atuação em equipes multiprofissionais. Em contrapartida, os concluintes classificaram de forma insuficiente as questões que envolveram o planejamento, a liderança e a administração e gerenciamento de serviços de saúde.

Quanto aos termos desconhecidos, ressalta-se a unanimidade da incompreensão para a expressão "alta pactuada", mesmo em estudantes no estágio final do curso. A alta pactuada em Saúde Bucal significa o compartilhamento das decisões terapêuticas entre profissional e paciente, sendo este último um sujeito capaz de autonomia ${ }^{55}$. Sugere-se que esse desconhecimento pode representar um modelo de relação profissional-paciente centrado no paciente ainda não culturalmente vivenciado por brasileiros ${ }^{56,57}$. Questiona-se a possível dificuldade no alcance das competências das DCN que preconizam a mudança desse modelo tanto na Atenção à Saúde, quanto nas práticas pedagógicas ${ }^{9,31}$. 
Outro aspecto a ser discutido nesse passo metodológico foi a satisfação dos estudantes quanto ao número de questões. Foram $41,2 \%$ que classificaram como regular, respondendo que "Sim, o número foi exagerado, mas achei importante a presença de todos os itens do questionário". Esse achado pode estar relacionado com viés de resposta associado a um questionário longo. Em contrapartida, o número de itens não deve obedecer a regras matemáticas, e, sim, a abrangência da teoria envolvida no construto $^{35}$.

O presente estudo culminou com a validação de uma escala composta por 53 itens e cinco indicadores, de acordo com critérios estabelecidos em recomendações nacionais e internacionais. Sugere-se a aplicação em diferentes cenários com teste de propriedades psicométricas e de forma longitudinal.

\section{CONCLUSÃO}

A EA-CO foi desenvolvida e atendeu aos critérios de validação de conteúdo e de face utilizados no estudo.

\begin{abstract}
Scale of attitudes related to dental competencies: development and validation

This study aimed to develop and validate the content and face of a scale of attitudes related to dental competencies (SA-DC). It was a methodological development study involving the design and validation of a scale, using a quantitative and qualitative approach. The theoretical framework for the construct of the scale involved the concept of attitude in the field of Social Psychology and that of competence in the field of Education, qualified by the National Curriculum Guidelines for Dentistry Programs, thus providing the following indicators: Empathy in the professional-patient relationship, Lifelong learning, Readiness for interprofessional education, Protection of patients' rights, Social
\end{abstract}

responsibility and Emotional intelligence. Ten experts participated in the face validation of the matrix of indicators (Nominal Group Technique) and of the items (Questionnaire), and the preliminary version was pilot tested with 17 undergraduate dentistry students. There was consensus on the permanence of the indicators, except for Emotional Intelligence. The scale (5point Likert type) resulted in 53 items, 13 of which were modified from the original version. SA-DC was developed and met the content and face validation criteria used in the study.

Descriptors: Education, Dental. Attitudes of Health Personnel. Professional Competence. Educational Measurement. Validation Studies.

\section{REFERÊNCIAS}

1. Myers, D G. Psicologia. Rio de Janeiro: LTC; 2012.

2. Brown G, Manogue M, Rohlin M. Assessing attitudes in dental education: is it worthwhile? Br Dent J. 2002;193(12):703-7.

3. Perrenoud P. Construir as competências desde a escola. Porto Alegre: Artmed; 1999.

4. Morin E. Os sete saberes necessários à educação do futuro. São Paulo: Cortez; 2000.

5. Lucchese, R, Barros,S. Pedagogia das competências - um referencial para a transição paradigmática no ensino de enfermagem - uma revisão da literatura. Acta Paul Enferm. 2006;19(1):92-9.

6. Merriënboer JJG, Kirschner PA. Ten Steps to Complex Learning. Reprinted (2009). New Jersey: Lawrence; 2007.

7. Brasil. Ministério da Educação (MEC). Conselho Nacional de Educação. Parecer CNE/CES n ${ }^{\circ} 116$ de 3 de abril de 2014. Diretrizes Curriculares Nacionais do Curso de Graduação em Medicina. Diário Oficial da União 2014; abr 3.

8. Brasil. Ministério da Saúde (MS). Conselho 
Nacional de Saúde. Resolução no 569 de 8 de dezembro de 2017. Diário Oficial da União 2018; 26 fev.

9. Brasil. Ministério da Educação (MEC). Conselho Nacional de Educação. Resolução $n^{\circ} 3$ de 19 de fevereiro de 2002. Diretrizes Curriculares Nacionais do Curso de Graduação em Odontologia. Diário Oficial da União 2002; 4 mar.

10. Field, JC, Cowpe, JG, Walmsley, AD. The Graduating European Dentist: A New Undergraduate Curriculum Framework. Eur J Dent Educ. 2017; 21(Suppl. 1):2-10.

11. American Dental Education Association (ADEA) House of Delegates. ADEA Competencies for the New General Dentist. J Dent Educ. 2011; 75(7): 932-5.

12. Ringsted C, Hodges B, Scherpbier A. 'The research compass': an introduction to research in medical education: AMEE Guide no. 56. Med Teach. 2011;33(9): 695-709.

13. Hojat M, Gonnella JS, Mangione1 S, Nasca TJ, Veloski JJ, Erdmann JB, Callahan CA, Magee M. Empathy in medical students as related to academic performance, clinical competence and gender. Med Educ. 2002; 36:522-7.

14. Nosow V, Püschel AVA. O ensino de conteúdos atitudinais na formação inicial do enfermeiro. Rev Esc Enf USP. 2009; 43(Esp2):1232-7.

15. Salgueira AP, Frada T, Aguiar P, Costa MJ. Aprendizagem ao longo da vida do médico: Tradução e Adaptação da Escala de Jefferson. Acta Med Port. 2009; 22:247-56.

16. Williams B, McCook F, Brown T, Palmero C, McKenna L, Boyle $\mathrm{M}$ et al. Are Undergraduate Health Care Students 'Ready' for Interprofessional Learning? A Cross-Sectional Attitudinal Study. IJAHSP. 2012;10(3):1-11.

17. Jahangiri L, Mucciolo TW, Choi M,
Spielman AI. Assessment of teaching effectiveness in U.S. Dental schools and the value of triangulation. $\mathrm{J}$ Dent Educ. 2008;72(6): 707-18.

18. Colares MFA, Troncon LEA, Figueiredo JF, Cianflone ARL, Rodrigues MLV, Piocinato $\mathrm{CE}$, et al. Construção de um instrumento para avaliação das atitudes de estudantes de Medicina frente a aspectos relevantes da prática médica. Rev Bras Educ Med. 2002;26(3):194-203.

19. Mascia AR, Silva FB, Lucchese AC, De Marco MA, Martins MCFN, Martins LAN. Atitudes frente a aspectos relevantes da prática médica: estudo transversal randomizado com alunos de segundo e sexto anos. Rev Bras Educ Med. 2009;33(1):41-8.

20. Miranda SM, Pires MMS, Nassar SM, Silva CAJ. Construção de uma escala para avaliar atitudes de estudantes de medicina. Rev Bras Educ Med. 2009;33(1)supl 1:104-10.

21. Andrade SC, Deus JA, Barbosa ECH, Trindade EMV. Avaliação do desenvolvimento de atitudes humanísticas na graduação médica. Rev Bras Educ Med. 2011;35(4): 517-25.

22. Paro HBMS, Daud-Gallotti, RM, Tibério IC, Pinto RMC, Martins MA. Brazilian version of the Jefferson Scale of Empathy: psychometric properties and factor analysis. BMC Med Educ. 2012;12:73.

23. Aguilar-da-Silva RH, Scapin LT, Batista NA. Avaliação da formação interprofissional no ensino superior em saúde: aspectos da colaboração e do trabalho em equipe. Avaliação (Campinas). 2011;16(1):165-84

24. Peduzzi M, Norman I, Coster S, Meireles E. Adaptação transcultural e validação da Readiness for Interprofessional Learning Scale no Brasil. Rev Esc Enferm USP. 2015; 49(2): 7-15. 
25. Brasil. Ministério da Educação (MEC). Secretaria de Educação Superior. Comissão Especial da Avaliação da Educação Superior (CEA). Sistema Nacional de Avaliação da Educação Superior SINAES: Bases para uma Nova proposta da Educação Superior. Brasília: Ministério da Educação; 2003. [Acesso em 28/12/2013]. Disponível em: http://www.unifesp.br/reitoria/orgaos/comis soes/avaliacao/sinaes.pdf.

26. Góes PSA, Fernandes LMA, Lucena LBS. Validação de Instrumentos de Coleta de Dados. In: Antunes JLF, Peres MA (eds). Fundamentos de Odontologia Epidemiologia da Saúde Bucal. Rio de Janeiro: Guanabara Koogan; 2006. p. 390-7.

27. Bezerra TCA, Falcão MLP, Goes PSA, Felisberto E. Avaliação de Programas de Formação Profissional em Saúde: construção e validação de indicadores. Trab Educ Saúde. 2016; 14(2): 445-72.

28. Jones J, Hunter D. Consensus methods for medical and health services research. BMJ. 1995; 311:376-80.

29. Streiner DL, Norman GR. Health Measurement Scales. 2 ed. New York: Oxford University Press; 1995.

30. Terwee CB, Bot SDM, Boer MR, van der Windt DAWM, Knol DL, Dekker J, Bouter LM, de Vet HCW. Quality criteria were proposed for measurement properties of health status questionnaires. J Clin Epidemiol. 2007;60(1): 34-42.

31. Brasil. Ministério da Saúde (MS). Diretrizes da política nacional de saúde bucal. Brasília: Ministério da Saúde; 2004.

32. Finkler M, Caetano JC, Ramos FRS. A dimensão ética da formação profissional em saúde: estudo de caso com cursos de graduação em odontologia. Cienc Saude Coletiva. 2011;16(11):481-92.

33. Brasil. Ministério da Saúde. Ministério da
Educação. A aderência dos cursos de graduação em enfermagem, medicina e odontologia às diretrizes curriculares nacionais. Brasília: Ministério da Saúde; 2006.

34. Morita MC, Kriger L, Carvalho ACP, Haddad AE. Implantação das diretrizes curriculares nacionais em odontologia. Maringá: Dental Press: ABENO: OPAS: MS: 2013.

35. Pasquali L. Testes referentes a construto: teoria e modelo de construção. In: Pasquali L, organizador. Instrumentação psicológica: fundamentos e prática. Porto Alegre: Artmed; 2010. p. 165-98

36. Mokkink A LB, Terwee CB, Patrick DL, Alonso J, Stratford PW, Knol DL, Bouoter $\mathrm{LM}$, de Vet HCW. International consensus on taxonomy, terminology, and definitions of measurement properties: results of the COSMIN study. J Clin Epidemiol. 2010; 63:737-45.

37. Mokkink LB, Terwee CB, Patrick DL, Alonso J, Stratford PW, Knol DL, Bouter LM, de Vet HCW. The COSMIN checklist for assessing the methodological quality of studies measurement properties of health status measurement instruments: an international Delphi study. Qual Life Res. 2010;(19):539-49.

38. Nicol DJ, Macfarlane-Dick D. Formative assessment and self-regulated learning: a model and seven principles of good feedback practice. Stud High Educ. 2006;31(2):199-218.

39. Norcini J, Anderson B, Bollela V, Burch V, Costa MJ, Duvivier R, Galbraith R, Hays R, Kent A, Perrott V, Roberts T. Criteria for good assessment: consensus statement and recommendations from the Ottawa 2010 Conference. Med Teach. 2011;33:206-14. 40. Borges MC, Miranda CH, Santana RC, 
Bollela VR. Avaliação formativa e feedback Como ferramenta de aprendizado na formação de profissionais da saúde. Medicina (Ribeirão Preto). 2014;47(3): 32431.

41. Morita MC, Scavuzzi AIF, Carcereri DL, Fontanella VRC. Documento orientador da ABENO para qualidade dos cursos de graduação em Odontologia. Rev ABENO. 2018; 18 (supl.2):1-38.

42. Alexandre NMC, Colusi MZO. Validade de conteúdo nos processos de construção e adaptação de instrumentos de medidas. Cienc Saude Coletiva. 2011; 16(7):3061-8.

43. Hojat M, Lanoue M. Exploration and confirmation of the latent variable structure of the Jefferson scale of empathy. Intern $\mathbf{J}$ Med Educ. 2014; 5:73-81.

44. Chiu C, Lu H, Arrigo LG, Wei CJ, Tsai D. A Professionalism Survey of Medical Students in Taiwan. J Exp Clin Med. 2010;2(1): 35-42.

45. Conselho Federal de Odontologia (CFO). Resolução n ${ }^{\circ}$. 118 de 11 maio de 2012. Código de Ética Odontológica. Rio de Janeiro: CFO; 2012.

46. Albino JEN, Young SK, Neumann LM, Kramer KA, Andrieu SC, Henson L et al. Assessing Dental Students' Competence: Best Practice Recommendations in the Performance Assessment Literature and Investigation of Current Practices in Predoctoral Dental Education. J Dent Educ. 2008;72(12):1405-35.

47. Braccialli LAD, Oliveira II, MAC. Desafios na Formação Médica: a Contribuição da Avaliação. Rev Bras Educ Med. 2012; 36(2) :280-8.

48. Hojat M, Veloski J, Nasca TJ, Erdmann JB, Gonnella JS. Assessing Physicians' Orientation Toward Lifelong Learning. JGIM. 2006:931-6.
49. World Health Organization (WHO). Health Professional Network Nursing and Midwifery. Department of Human Resources for Health. Framework for Action on Interprofessional Education \& Collaborative Practice. Geneva: WHO/HRH/HPN/10.3; 2010.

50. Thannhauser J, Russell-Mayhew S, Scott C. Measures of interprofessional education and collaboration. J Interprof Care. 2010;24(4): 336-49.

51. Brasil. Ministério da Educação (MEC). Conselho Nacional de Educação. Câmara de Educação Superior. Resolução $N^{\circ} 7$ de 18 de dezembro de 2018. Diretrizes para a Extensão na Educação Superior Brasileira. Brasília: Ministério da Educação; 2018

52. Arora S, Ashrafian H, Davis R, Athanasiou T, Darzi A, Sevdalis N. Emotional intelligence in medicine: a systematic review through the context of the ACGME competencies. Med Educ. 2010;44:749-64.

53. Bueno JMH, Correia FMDL, Gomes MAYA, Júnior FSP. Competências emocionais: estudo de validação de um instrumento de medida. Aval Psicol. 2015; 14(1):153-63.

54. Fadel CB, Baldani MH. Percepções de formandos do curso de odontologia sobre as diretrizes curriculares nacionais. Trab Educ Saúde. 2013;11(2):339-54.

55. Pezzato LM, L'Abbate S. Uma PesquisaAção-Intervenção em Saúde Bucal Coletiva: contribuindo para a produção de novas analyses. Saúde Soc. 2012; 21(2):386-98.

56. Ribeiro MMF, Krupat E, Amaral CFS. Brazilian medical students' attitudes towards patient-centered care. Med Teach. 2007;29:(6):e204-8.

57. Mota LDQ, Santos TAD, Magalhães DBL. Humanização no atendimento odontológico: acolhimento da subjetividade dos pacientes 
atendidos por alunos de graduação nos campos de estágio. RBCS. 2012;16(4): 53744.

58. Hojat M, Mangione S, Nasca TJ, Cohen MJM, Gonnella JS, Erdmann JB, et al. The Jefferson Scale of Physician Empathy: Development and preliminary psychometrics. Educ Psychol Meas. 2001;61:349-65.

59. Silverman J, Kurtz S, Draper J. Skills for Comunicating with Patients. Oxford: Radcliffe; 2005.

60. Brasil. Ministério da Saúde (MS). Secretaria de Atenção à Saúde. Núcleo Técnico da Política Nacional de Humanização. Clínica ampliada, equipe de referência e projeto terapêutico singular. Brasília: Ministério da Saúde [Série B. Textos Básicos de Saúde]; 2008.

61. Parsell J, Bligh G. The development of a questionnaire to assess the readiness of health care students for interprofessional learning (RIPLS). Med Educ. 1999; 33:95100.

\section{Correspondência para:}

Manoela Almeida Santos da Figueira e-mail: mf@fps.edu.br; manufigueira@hotmail.com Avenida Mal. Mascarenhas de Morais, 4861, Imbiribeira 51.150-000 Recife/PE 